\title{
Generating Test Data for Specification-Based Tests Via Quasirandom Sequences
}

\author{
Hongmei Chi ${ }^{1,2}$, Edward L. Jones ${ }^{2}$, Deidre W. Evans ${ }^{2}$, and Martin Brown ${ }^{2}$ \\ 1 School of Computational Science, \\ Florida State University, Tallahassee, FL 32306-4120 \\ chi@csit.fsu.edu \\ 2 Department of Computer and Information Sciences, \\ Florida A\& M University, Tallahassee, FL 32307-5100
}

\begin{abstract}
This paper presents work on generation of specificationdriven test data, by introducing techniques based on a subset of quasirandom sequences (completely uniformly distributed sequences) to generate test data. This approach is novel in software testing. This enhanced uniformity of quasirandom sequences leads to faster generation of test data covering all possibilities. We demonstrate by examples that welldistributed sequences can be a viable alternative to pseudorandom numbers in generating test data. In this paper, we present a method that can generate test data from a decision table specification more effectively via quasirandom numbers. Analysis of a simple problem in this paper shows that quasirandom sequences achieve better data than pseudorandom numbers, and have the potential to converge faster and so reduce the computational burden. Functional test coverage, an objective criteria, evaluates the quality of a test set to ensure that all specified behaviors will be exercised by the test data.
\end{abstract}

Keywords: automatic test case generation, specification-driven test, functional test coverage, quasirandom numbers, well-distributed sequences.

\section{Introduction}

Software testing [14 is a costly process that is critical for accessing system behavior. The two common strategies are black-box testing, driven by specification of software 22], and white box testing, driven by the software structure 16. Specification-based testing, whose inputs are derived from a specification, is black-box testing. Specification-based testing of software is to increase the effectiveness of software testing [13. A formal software specification is one of the most useful documents to have when testing software, since it is a concise and precise description of functionality. Specification-based testing focuses on obtaining test data from specification [19. Generating test data to cover all specification is a challenge for a complex system [6,21.

We are developing an approach to deriving test data from quasirandom sequences [20] instead of pseudorandom sequences. Quasirandom sequences are 
constructed to minimize the discrepancy, a measure of the deviation from uniformity and therefore quasirandom sequences are more uniformly distributed than pseudorandom sequences. In the past, pseudorandom number generators, such as linear congruential generators [9] have been used in the implementation of random testing. Recently, it has been recognized that the convergence rate of Monte Carlo approaches based on pseudorandom numbers is slow and that an important improvement of the convergence rate can be achieved by using quasi-Monte Carlo methods [12,15]. This observation is the motivation for the investigation described in this paper.

We will explore the use of completely uniformly distributed sequences in generating test data. The organization of this paper is following. An overview of completely uniformly sequences (quasirandom sequences) is given in $\S 2$. An overview for specification-based tests and test data generation is presented in $\S$ 3 . In $\S 4$, we analyze a simple specification-based test problem using completely uniformly sequences and numerical results are shown. A discussion of results and conclusion are presented in $\S 5$.

\section{Quasirandom Sequences}

Pseudorandom numbers are constructed to mimic the behavior of truly random numbers, whereas highly uniform numbers, called quasirandom numbers, are constructed to be as evenly distributed as is mathematically possible. Pseudorandom numbers are scrutinized via batteries of statistical tests that check for statistical independence in a variety of ways, and are also checked for uniformity of distribution, but not with excessively stringent requirements. Thus, one can think of computational random numbers as either those that possess considerable independence, such as pseudorandom numbers; or those that possess considerable uniformity, such as quasirandom numbers [15].

From Fig. 1, we can see the difference between pseudorandom and quasirandom sequences. Pseudorandom numbers are only a subsitute for true random numbers and tend to show clustering effects; while quasirandom numbers tends to more uniformly distributed. There are many applications that do not really require randomness, but instead require numbers that uniformly cover the sample space. Quasirandom sequences are more suitable for such applications. In particular, fewer quasi-random samples are needed to achieve a similar level of accuracy as obtained by using pseudo-random sequences [11, 18.

The original construction of quasirandom sequences is related to the Weyl sequence [10 and the van der Corput sequence. Weyl sequence is based on irrational numbers while the van der Corput sequence is a one-dimension quasirandom sequence based on digital inversion. This digital inversion method is the central idea behind the construction of current quasirandom sequences, such as Halton, Faure and Sobí sequences. Niederreiter [15] extended this method to arbitrary bases and dimensions. Weyl sequences is used this paper for numerical experiments in $\S 4$. The definition of Weyl sequence is as follows: 
Definition 1. If $\theta$ is an irrational number, then the Weyl sequence $n * \theta$ $(\bmod 1), n=1,2,3, \ldots$, is uniformly distributed.

Here $(\bmod 1)$ is operation of keeping the fraction part of any number, for example $2.345(\bmod 1)=0.345$. The Weyl sequence is easy to implement and well-distributed. For different $\theta$, the different dimensions of the Weyl sequence can be generated.
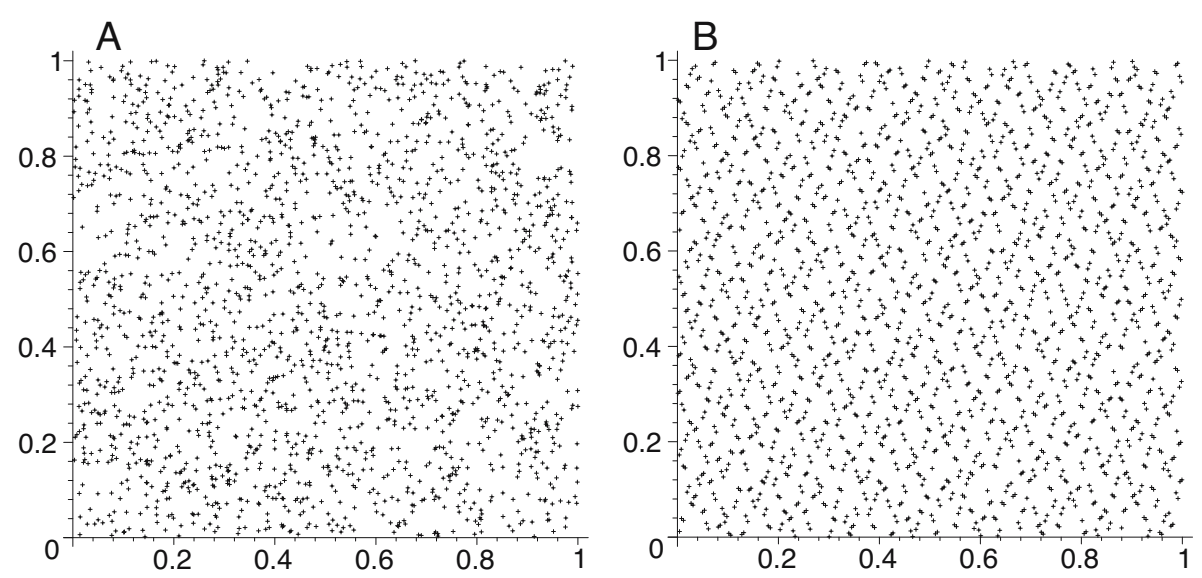

Fig. 1. Comparison of pseudorandom numbers and quasirandom numbers in two dimensions. A: 2000 pseudorandom numbers (linear congruential generator); B: 2000 quasirandom numbers (Weyl sequence).

\section{Specification-Based Tests}

Although a formal software specification is one of the most useful document to have when testing software, most of software specifications are stated informally in practice and that leaves a lot of ambiguities. Additional specification notations are needed to clarify these statements. A decision table is a rule-based specification in which responses are specified in terms of combinations of conditions met by input data. The decision table is a specification technique that can be used as the basis for test case design [3, 5, 8]. In this section we show by an example how a decision table can provide the basis for defining specification-based tests. We also show that how quasirandom sequences produce the test data based on the decision table.

One measure for test case effectiveness is defined as functional coverage, which measures the thoroughness of testing based on the specification. This is a ratio of the number of rules triggered by the set of test data to the number of rules in the decision table.

Definition 2. Functional coverage $=\frac{\# \text { rules-satisfied }}{\# \text { rules-in-the-decision-table }}$. 


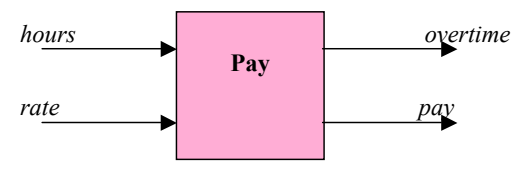

Fig. 2. Black-box schematic specification for Pay

\begin{tabular}{|c|c|}
\hline CONDITIONS & I DECISION RULES \\
\hline hours $>40$ & $\begin{array}{lllll}\text { I } & N & Y & N & Y\end{array}$ \\
\hline rate $<10$ & I Y Y N N \\
\hline ACTIONS & I ACTION RULES \\
\hline pay $=$ hours $*$ rate $;$ pay $=$ pay & $1 \quad x---$ \\
\hline pay $=1.5 *$ rate $*($ hours -40$)+40 *$ rate; & $1-x--$ \\
\hline pay $=40 *$ rate; & $1--x x$ \\
\hline overtime $=0$ & i $x-x x$ \\
\hline overtime $=1.5 *$ rate $*($ hours -40$)$; & $-x--$ \\
\hline
\end{tabular}

Fig. 3. Payroll Decision Table (DT1) based on the Narrative Specification in Table 1

Consider the narrative specification in Table 1 [7, which specifies software to compute the weekly pay of employees. The first step in transforming this narrative specification is to identify the stimuli and responses. From the specification, we can deduce that the necessary stimuli (input data) are the hours worked and the hourly salary rate. The responses are the amount of the pay and the resulting overtime paid as shown in Figure 2. According to the specification, the software must determine whether an employee is hourly (rate $\leq 10$ ) or salaried, and whether the employee has exceeded 40 hours of work (hours $\geq 40$ ). Figure 3 is a summary of all rules and actions for Pay Specification in Table 1.

Table 1. Payroll Specification

Calculate employee pay, including overtime paid at 1.5 times the hourly
rate of hourly employees for time in excess of 40 hours. Salaried employees
are not paid overtime, nor do they lose pay when they work less than the
normal work week of 40 hours. Hourly employees earn less than 10 per hour.

When testing complicated software with a static specification, it is difficult to determine manually whether each rule has been covered and if there are anomalies in the decision table specification [4. Jones [7] 8] has developed a tool that uses test data to identify specification anomalies, while using the specification to determine adequacy of the test data. We use quasirandom sequences to provide the test data, and functional coverage as the criterion for measuring the test data. The procedure is simple: according Figure 2, we generate two-dimension 
Table 2. Payroll Specification (Extended from Table 1)

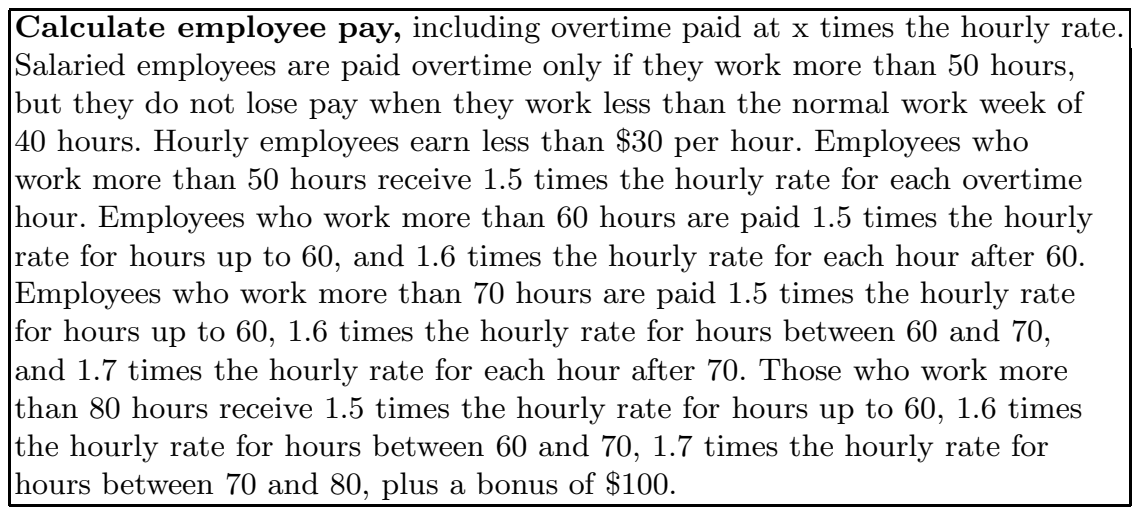

\begin{tabular}{|c|c|c|c|c|c|c|c|c|}
\hline Conditions & \multicolumn{8}{|c|}{ Decision Rules } \\
\hline hours $>40$ & $\mathrm{~N}$ & $\mathrm{~N}$ & $\mathrm{Y}$ & $\mathrm{Y}$ & & & & \\
\hline hours $>50$ & & & & & $Y$ & & & \\
\hline hours $>60$ & & & & & & $\bar{Y}$ & & \\
\hline hours $>70$ & & & & & & & $Y$ & \\
\hline hours $>80$ & & & & & & & & $\mathrm{Y}$ \\
\hline rate $>=30$ & $\mathrm{~N}$ & $\bar{Y}$ & $\mathrm{~N}$ & $\mathrm{Y}$ & $\mathrm{Y}$ & $\bar{Y}$ & $\mathrm{Y}$ & $\mathrm{Y}$ \\
\hline Actions & \multicolumn{8}{|c|}{ Action Rules } \\
\hline regular_pay $=$ hours ${ }^{*}$ rate & $x$ & & & & & & & \\
\hline regular_pay $=40 *$ rate & & $x$ & $x$ & $x$ & $x$ & $\mathrm{x}$ & $x$ & $x$ \\
\hline over_pay $=0$ & $x$ & $x$ & & & & & & \\
\hline over_pay $=1.5 *$ rate * $($ hours -40$)$ & & & $x$ & & $x$ & & & \\
\hline $\begin{array}{c}\text { over_pay }=1.5^{*} \text { rate * }(20)+1.6 \text { * } \\
\text { rate * }(\text { hours }-60)\end{array}$ & & & & & & $x$ & & \\
\hline $\begin{array}{c}\text { over_pay }=1.5^{*} \text { rate * }(20)+1.6^{*} \\
\text { rate * }(10)+1.7^{*} \text { rate }^{*}(\text { hours - } \\
70)\end{array}$ & & & & & & & $x$ & \\
\hline $\begin{array}{l}\text { over_pay }=1.5^{*} \text { rate }{ }^{*}(20)+1.6 * \\
\text { rate }^{*}(10)+1.7^{*} \text { rate } *(10)+100\end{array}$ & & & & & & & & $x$ \\
\hline
\end{tabular}

Fig. 4. Payroll Decision Table 2 (DT2) based on the Narrative Specification in Table2

Table 3. Test Results for Decision Tables (\#test data for full funtional coverage)

\begin{tabular}{|c|cr|rc|}
\hline Generator & \multicolumn{2}{|c|}{ Decision Table DT1 } & \multicolumn{2}{c|}{ Decision Table DT 2 } \\
\cline { 2 - 4 } & \#rules & \#test data pairs & \#rules & \#test data pairs \\
\hline \hline PRNG & 4 & 6 & 8 & 29 \\
\hline QRNG & 4 & 5 & 8 & 11 \\
\hline
\end{tabular}


Table 4. Test data Generated by a QRNG for Decision Tables DT 1 and DT 2

\begin{tabular}{|c|c|c|}
\hline & Test Data for DT 1 & Test Data DT 2 \\
\hline \# DT rules & 4 & 8 \\
\hline \# test pairs & 5 & 11 \\
\hline hours & \begin{tabular}{lllll|}
48 & 58 & 25 & 29 & 70 \\
\end{tabular} & $\begin{array}{llllllllll}88 & 76 & 43 & 110 & 36 & 62 & 67 & 51 & 15 & 78 \\
\end{array}$ \\
\hline rate & $\begin{array}{lllll}15 & 13 & 8 & 19 & 6\end{array}$ & $\begin{array}{lllllllllll}16 & 40 & 32 & 52 & 40 & 56 & 40 & 51 & 31 & 38 & 13\end{array}$ \\
\hline rule & $\begin{array}{lll}2 & 2 & 3\end{array}$ & $\begin{array}{llllll}3 & 5 & 7 & 4 & 2 & 6\end{array}$ \\
\hline Uncovered rules & none & none \\
\hline
\end{tabular}

Table 5. Test data Generated by a PRNG for DecisionTables DT 1 and DT 2

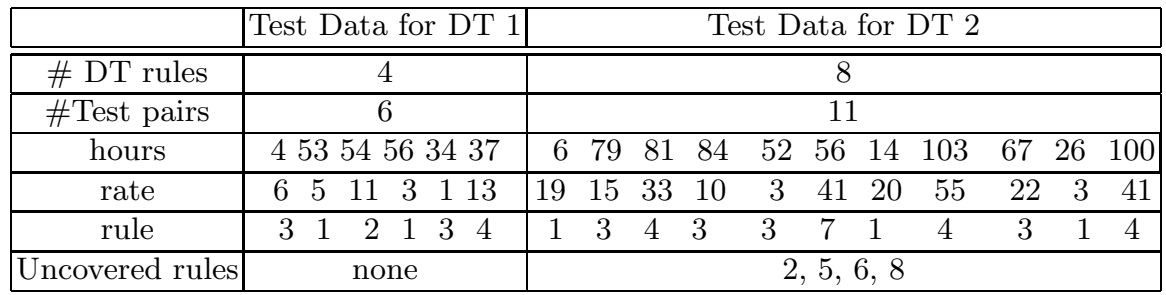

test data sets (hour, rate), and check functional coverage to see how many decision table rules are satisified (covered) by one or more test data pairs. The measure of interest for comparing pseudo-random and quasirandom generation of data sets is the number of test data needed to reach functional coverage of 1 . The numerical results are shown in Section 4.

\section{Numerical Experiments}

We need a more complicated decision table for generating test data. Therefore, we extend the decision table ( in Table 1 and Figure 3) and make more rules. The new specification narrative and decision table are shown in Table 2 and Figure 4, respectively.

In order to compare the effectiveness of quasirandom numbers, we use quasirandom sequences and pseudorandom sequences to produce the test data. The numerical results are shown in Table 2. The pseudorandom number generator(PRNG) we used in this paper is one of linear congruential generators (LCGs) in Numerical Recipe in C [17]. This LCG is defined as following:

Definition 3. The $L C G$ determined by $x_{n}=a x_{n-1}(\bmod m)$ with $a=16807$ and $m=2^{31}-1$ has a period of $2^{31}-2$.

The quasirandom number generator is Weyl sequences and we used the same implementation in [2]. The Weyl sequence we used in this paper with $\theta=2$. The results in Table 3 show that quasirandom number generator(QRNG) significantly converges faster, i.e., covers all rules with fewer test data. The quasirandom test 
data are presented in Table 4, the pseudorandom test data in Table 5. The faster convergence is more marked for the large decision table.

\section{Conclusions and Future Work}

A new scheme for generating test data via quasirandom sequences is proposed. The advantage of this scheme is that we can provide test data based on a specification automatically. This scheme is an alternative to generate test data manually or from pseudorandom numbers. Our numerical results, though preliminary , are promising. Should our observations about faster convergence (full coverage with fewer test data) hold, quasirandom test generation may offer economical advantages over pseudo-random testing. A broader question is whether quasirandom testing is superior to pseudo-random testing, in terms of efficiency and effectiveness. Addressing this question may require a replication of past studies such as in Abdurazik 1].

In the future, we will extend the study given in this paper to support the test-driven specification paradigm of Jones [7, 8] when applied to more complex problems requiring large, complex decision table specifications. Ongoing work includes the development of a library of quasirandom generation routines to support specification-based test generation.

One of the limitations of this scheme may occur when input is extremely distributed instead of uniformly distributed. On the other hand, because many of the accepted software testing practices are based on partitioning and sampling, the impact of non-uniform distributions may be negligible.

\section{References}

1. A. Abdurazik, P. Ammann, W. Ding, and J. Offutt. Evaluation of three specification-based testing criteria. Sixth IEEE International Conference on Complex Computer Systems (ICECCS'00), pages 179-187, 2000.

2. P. Beerli, H. Chi, and E. Jones. Quasi-monte carlo method in population genetics parameter estimation. Mathematics and Computers in Simulation, In press, 2006.

3. R. V. Binder. Testing Object-oriented systems: models, patterns and tools. AddisonWesley, Reading, Massachusetts, 1999.

4. K. H. Chang, S. Liao, and R. Chapman. Test scenario geneneration based on formal specification and usage. International Journal of Software Engineering and Knowledge Engineering, 10(2):1-17, 2000.

5. N. Glora, H. Pu, and W. O. Rom. Evaluation of process tools in systems analysis. Information and Technology, 37:1191-1126, 1995.

6. J. B. Goodenough and S. L. Gerhart. Toward a theory of test data selection. In Proceedings of the international conference on Reliable software, pages 493-510, 1975.

7. E. L. Jones. Automated support for test-driven specification. In Proceedings of the 9th IASTED International Conference on Software Engineering and Applications, pages 218-223, 2005.

8. E. L. Jones. Test-driven specification: Paradigm and automation. In 44th ACM Southeast Conference, March 10-12, 2006. 
9. D. E. Knuth. The Art of Computer Programming, vol. 2: Seminumerical Algorithms. Cambridge University Press, New York, 1997.

10. L. Kuipers and H. Niederreiter. Uniform Distribution of Sequences. John Wiley and Sons, New York, 1974.

11. W. L. Loh. On the asymptotic distribution of scrambled net quadrature. Annals of Statistics, 31:1282-1324, 2003.

12. W.J. Morokoff and R.E. Caflish. Quasirandom sequences and their discrepancy. SIAM Journal on Scientific Computing, 15:1251-1279, 1994.

13. H. Muccini, A. Bertolino, and P. Inverardi. Using software architecture for code testing. IEEE Trans. on Software Engineering, 30(3):160-171, 2004.

14. G. Myers, C. Sandler, T. Badgett, and T. Thomas. The Art of Software Testing. John Wiley and Sons, New Jersey, 2004.

15. H. Niederreiter. Random Number Generations and Quasi-Monte Carlo Methods. SIAM, Philadelphia, 1992.

16. S. Ntafos. A comparison of some structural testing strategies. IEEE Trans. on Software Engineering, 14(6):868-874, 1988.

17. W. H. Press, S. A. Teukolsky, W. T. Vetterling, and B.P. Flannery. Numerical Recipes in C. Addison-Wesley, Reading, Massachusetts, 1992.

18. J. Spanier and E. Maize. Quasirandom methods for estimating integrals using relatively small sampling. SIAM Review, 36:18-44, 1994.

19. P. Stocks and D. Carrington. Test template framework: A specification-based testing case study. In Proceedings of Int. Symp. Software Testing and Analysis, pages 11-18, 1993.

20. S. Tezuka. Uniform Random Numbers, Theory and Practice. Kluwer Academic Publishers, IBM Japan, 1995.

21. P. Variyam. Specification-driven test generation for analog circuits. IEEE Trans. on Computer-aided Design, 19(10):1189-1201, 2000.

22. G. Wimmel, H. Ltzbeyer, A. Pretschner, and O. Slotosch. Specification based test sequence generation with propositional logic. Software Testing, Verification and Reliability, 10(4):229-248, 2000. 